\title{
Clinical and laboratory profile of Scrub typhus in paediatric age group
}

\author{
Atindra Mishra', Rupa Thakur ${ }^{2}$, Ruby Thakur ${ }^{1}$ \\ ${ }^{1}$ Lecturer, Department of Paediatrics, National Medical College, Birgunj, Nepal, '2Lecturer, Department of Dermatology, \\ National Medical College, Birgunj, Nepal
}

Background: Scrub typhus is an acute febrile illness caused by Orientia tsutsugamushi, obligate intracellular organism. Incidence of Scrub typhus is increasing due to easy availability and decreased cost of diagnostic facility. This study was designed to study the clinical and laboratory profile of scrub typhus patient in department of paediatrics at National Medical College, Birgunj. Aims and Objectives: To study the clinical manifestations, laboratory findings and complications of Scrub typhus in Pediatric age group. Methods and Methods: A prospective observational study was carried out in children below the age of 15 years, admitted in paediatrics department of a tertiary care hospital of Nepal from June 2019 to May 2020. Clinical manifestations and laboratory findings were collected and recorded in predesigned data sheet. Scrub typhus was diagnosed with IgM ELISA. Results: A total of 52 patients were diagnosed as scrub typhus. All patients presented with fever and commonly had other symptoms such as headache $(65.3 \%)$, vomiting $(63.5 \%)$, cough $(59.6 \%)$, breathing difficulty $(57.7 \%)$, abdominal pain $(53.9 \%)$, seizures $(21.2 \%)$ and rashes $(9.6 \%)$. Important clinical signs noticed were lymphadenopathy $(84.6 \%)$, edema $(76.4 \%)$, hepatomegaly $(56.8 \%)$, splenomegaly $(36.5 \%)$ jaundice $(30.7 \%)$. About $12 \%(n=9)$ had necrotic eschar. $54 \%$ of the admitted patient confirmed using open defecation. Myocarditis $(67.3 \%)$ was the commonest complication followed by Acute Kidney Injury (65.4\%). Conclusions: Scrub typhus must be considered in differential of tropical fever in children, especially in those residing in rural area and having open defecation. In our study, Fever was the ubiquitous symptom and Myocarditis was the commonest complication.

Key words: Capillary leak; Fever Lymphadenopathy; Myocarditis; Scrub typhus
Access this article online

Website:

http://nepjol.info/index.php/AJMS

DOI: 10.3126/ajms.v11i6.29913

E-ISSN: 2091-0576

P-ISSN: 2467-9100

Copyright (c) 2020 Asian Journal of Medical Sciences

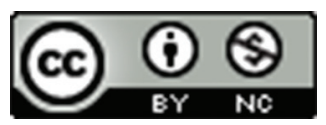

This work is licensed under a Creative Commons Attribution-NonCommercial 4.0 International License.

\section{INTRODUCTION}

Scrub typhus is an acute febrile illness caused by Orientia tsutsugamushi, a zoonotic bacterial infection, transmitted by bite of trombuculid mite. ${ }^{1}$ Human acquires the disease from the bite of an infected chigger. ${ }^{2}$ Scrub typhus is underdiagnosed in our part of the world as index of suspicion is low among the paediatricians. ${ }^{3}$ Nonspecific clinical signs and symptoms, limited awareness about the disease and unavailability of diagnostic facilities are the reasons for underdiagnosis of the disease. Delay in diagnosis and treatment have been associated with increase in case fatality. Scrub typhus in Nepal was first ever reported in 1981 and a hospital-based study carried out in 2004 found 28 cases of scrub typhus among 876 enrolled febrile patients. ${ }^{4}$
Scrub typhus is often acquired during occupational/ agricultural exposures because active rice fields are an important reservoir for transmission. Children playing bare foot in the foothills, transmission during open defecation are risk factors. ${ }^{5}$ The incubation period of scrub typhus is 5-20 days and has distinct eschar at the site of chigger bite, helpful in making diagnosis. Clinical manifestations include fever, headache, hepatomegaly, splenomegaly and complications include Acute Kidney Injury (AKI), Myocarditis, Meningitis, Disseminated Intravascular Coagulation (DIC). Scrub typhus should be considered as a differential for undifferentiated fever. ${ }^{6}$

The data regarding the scrub typhus in children is scarce from Nepal. Hence, this study was planned prospectively 
to study the clinical and laboratory profile of scrub typhus in children less than 15 years.

\section{MATERIALS AND METHODS}

\section{Study design}

Hospital based prospective observational study done in department of paediatrics from June 2019 to May 2020.

\section{Ethical approval}

The study was approved by the Institute Research Committee (IRC) of National Medical College, Birgunj, Nepal. Written and oral consents were taken from the informants.

\section{Participants and settings}

All patients below the age of 15 years with Immunoglobulin M Enzyme linked Immunosorbent Assay (IgM ELISA) positivity for Scrub typhus and admitted in Pediatrics Ward/ Pediatric Intensive Care Unit (PICU) of National Medical College and Teaching Hospital (NMCTH), Birgunj were enrolled in the study. The IgM antibody to Orientia tsutsugamushi was detected by using Scrub Typhus Detect ${ }^{\mathrm{TM}}$ Kit, In Bios International, USA, and the optical density was measured by Huma Reader HS, ELISA reader, with optical density (OD) $>0.50$ being considered positive.

\section{Inclusion criteria}

All children diagnosed as Scrub Typhus with IgM ELISA for Scrub Typhus.

\section{Exclusion criteria}

Those patients with proven co-infection and clinically suspected Scrub Typhus without IgM ELISA positivity.

\section{Definitions}

\section{Acute kidney injury (AKI)}

Rise in serum creatinine of at least $0.3 \mathrm{mg} / \mathrm{dl}$ or $50 \%$ higher than baseline within a $24-48$ hour period or a reduction in urine output to $0.5 \mathrm{ml} / \mathrm{kg}$ per hour for longer than 6 hours.

\section{Meningoencephalitis}

Altered sensorium along with signs of meningeal irritation and/or seizures associated with elevated protein and lymphocytic / neutrophilic cytology with normal or low sugar on CSF analysis.

\section{Myocarditis}

Child with tachypnoea, tachycardia and/or (S3-gallop, shock) along with echocardiographic finding suggestive of reduced ejection fraction and elevated cardiac enzymes (CK- MB)

\section{Hypoalbuminemia}

Serum Albumin level less than $2.5 \mathrm{gm} / \mathrm{dl}$

\section{IgM ELISA for scrub typhus}

The IgM antibody to Orientia tsutsugamushi was detected by using Scrub Typhus Detect ${ }^{\mathrm{TM}}$ Kit, In Bios International, USA, and the optical density was measured by HumaReader HS, ELISA reader, with optical density (OD) $>0.50$ being considered positive. The cut-off was calculated following recommendations for determining the endemic cut-off titre in the kit protocol.

\section{Variables}

Clinical data, fever defervescence, associated symptoms, vital signs, and the general and systemic examination findings, were recorded. A careful search for eschar was performed in all patients. Data regarding age, sex and residential area, outdoor activity were collected. Complete blood counts, chest X-rays, urea, creatinine, aspartate transaminase, alanine transaminase, serum albumin, were performed at the time of presentation for all cases and were repeated if necessary.

\section{Data Sources/Measurement}

A predesigned proforma were filled to include the history and clinical findings. The data were analysed using MS Excel 2010, and SPSS version 20.0. Descriptive statistics in terms of frequency, percentage, mean, and standard deviation were calculated.

\section{RESULTS}

A total of 52 patients with clinical features suggestive of Scrub typhus and those who tested positive for IgM ELISA for Scrub Typhus were included in this study. All children below 15 years were included in the study with mean age of 8.3 years. Majority, 34 children $(65.4 \%)$ were male with male female ratio of 1.8:1. The study was conducted in Parsa district of Nepal, majority of the admitted children were from bordering district of Bara, followed by Rauthat and Sarlahi district.

Fever was present in all children and 26 children $(50 \%)$ had fever of more than 7 days duration. Fever defervescence was achieved after 2.1 day of treatment. Common symptoms in children were headache 34 (65.3\%), vomiting $33(63.5 \%)$, abdominal pain $28(53.9 \%)$, cough 31 (59.6\%), difficulty in breathing $30(57.7 \%)$ respectively. Important clinical signs noticed on examination were lymphadenopathy 44 (84.6\%), hepatomegaly $29(56.8 \%)$, edema $40(76.9 \%)$, jaundice 16 (30.7\%), splenomegaly $19(36.5 \%)$. Eschar was present in $6(11.5 \%)$ children. Table 1 shows the clinical profile of children at the time of admission.

Thrombocytopenia was the prominent laboratory finding seen in $47(90.4 \%)$ children. Nine $(17.3 \%)$

Asian Journal of Medical Sciences | Nov-Dec 2020 | Vol 11 | Issue 6 
children had platelet count less than $50000 / \mathrm{mm}^{3}$ and $29(55.7 \%)$ children had between platelet between $50000 / \mathrm{mm}^{3}$ to $1 \mathrm{lakh} / \mathrm{mm}^{3}$. Forty-three (82.6\%) children had Hypoalbuminemia (Albumin $<2.5 \mathrm{~g} / \mathrm{dl}$ ) with average serum albumin of $2.1 \mathrm{~g} / \mathrm{dl}$. Raised liver enzymes of more than 2 times were seen in $42(80.7 \%$ ) children with average AST/ALT of 112IU/L and 120IU/L respectively. Table 2 depict the laboratory parameters of the children with scrub typhus.

Myocarditis was the commonest complication seen in $35(67.3 \%)$ children followed by Acute Kidney Injury (AKI) seen in $34(65.4 \%)$ children. Meningoencephalitis was seen in 8 children (15.4\%), 6 children had MODS. Only 2 children in MODS survived while 4 succumbed to death (Table 3). In our study, 44 (84.6\%) children were treated with Doxycycline alone, while the remaining were treated with Doxycycline and Azithromycin. Four children succumbed to death with mortality rate of $7.7 \%$.

Our study found open defecation in field or movement of children in forest area/rice field as the risk factor for scrub typhus disease. 28 (54\%) children confirmed open defecation, while outdoor play in nearby forest and area with heavy vegetation was seen in $6(11.5 \%)$ children. Table 4 shows risk factor for contracting scrub typhus in children.

\begin{tabular}{lcc}
$\begin{array}{l}\text { Table1: Clinical manifestations of patients with } \\
\text { scrub typhus }\end{array}$ & Number & Percentage \\
\hline Symptoms/Signs & 52 & \\
\hline Duration of fever $(\mathrm{N}=52)$ & 09 & 17.4 \\
1-3 days & 17 & 32.6 \\
3-7 days & 26 & 50 \\
$>7$ days & 44 & 84.6 \\
Lymphadenopathy & 40 & 76.9 \\
Edema & 34 & 65.3 \\
Headache & 33 & 63.5 \\
Vomiting & 31 & 59.6 \\
Cough & 30 & 57.7 \\
Breathing difficulty & 29 & 56.8 \\
Hepatomegaly & 28 & 53.9 \\
Abdominal pain & 19 & 36.5 \\
Splenomegaly & 16 & 30.7 \\
Jaundice & 11 & 21.2 \\
Seizure & 6 & 11.5 \\
Eschar & 5 & 9.6 \\
Rash & & \\
\hline
\end{tabular}

\begin{tabular}{lc} 
Table 2: Laboratory parameters in patient with \\
Scrub Typhus \\
\hline Lab parameters & $\mathbf{N}=\mathbf{5 2}$ \\
\hline Leucocyte count & $8000(3600-14600)$ \\
AST & 112 \\
ALT & 120 \\
Serum Albumin & 2.1 \\
Thrombocytopenia & $80,000(14,000$ to 180,000$)$ \\
\hline
\end{tabular}

\section{DISCUSSION}

This observational study was done at tertiary care centre and children below the age of 15 years with scrub typhus positivity (IgM ELISA) were enrolled in the study. Scrub typhus occurs after bite of thromboculid mite and manifests as undifferentiated fever. ${ }^{3}$

Our study showed Scrub typhus to be common in male $65.4 \%$, similar to study from Taiwan $60.7 \%$, probably because males remain outdoor longer while playing and gets indulged in agricultural activities. ${ }^{7}$

The mean age of children in our study was 8.3 years. Mean age of 8.8 years was observed by both Bhat et al from India and Pathak et al from Nepal. ${ }^{8,9}$ The incidence of scrub typhus increases with age as older children remains outdoor compared to younger. ${ }^{3}$

Scrub typhus has been on rise in recent past and outbreaks have been reported from various parts of the country. ${ }^{10}$ Most of these cases were seen during the months of July to November. Post monsoon surge in disease has been reported by different authors. ${ }^{11,12}$ More cases were seen during these months because children from rural areas often gets involved in fields, where they are exposed to the bites of larval mites.

Scrub typhus has varied clinical presentation, with fever being the most common symptom. ${ }^{8}, 13$ In our study, fever was documented in all patients with, $26(50 \%)$ of the children having fever of less than 7 days duration. Kumar et al and Palanivel et al also demonstrated fever to be present in all cases. ${ }^{8,13}$ Fever defervescence occurred after 2.1 days of treatment with doxycycline and/or Azithromycin. Similarly, studies from Taiwan showed fever defervescence in 2.8 days when treated with Doxycycline. ${ }^{7}$

\begin{tabular}{lcc}
\multicolumn{3}{l}{ Table 3: Complications in scrub patients } \\
\hline Complication & Frequency (N) & Percentage (\%) \\
\hline Myocarditis & 35 & 67.3 \\
Acute kidney Injury (AKI) & 34 & 65.4 \\
Severe thrombocytopenia & 9 & 17.3 \\
Meningoencephalitis & 8 & 15.4 \\
MODS & 6 & 11.5 \\
\hline
\end{tabular}

\begin{tabular}{lcc} 
Table 4: Risk factor for contracting Scrub Typhus \\
\hline Risk factor & Frequency & Percentage (\%) \\
\hline Open defecation & 28 & 54 \\
Outdoor playing (nearby forest/ & 6 & 11.5 \\
vegetation) & & \\
Forest work & 5 & 10 \\
Unidentified & 13 & 24.5 \\
Total & 52 & 100 \\
\hline
\end{tabular}


Another study had average of 29 hours interval to fever defervescence after treatment. ${ }^{12}$

Children with scrub typhus often presents with fever and generalised body swelling. ${ }^{1}$ Capillary leak and Hypoalbuminemia causes body swelling. O. tsutsugamushi infects vascular endothelium leading to vasculitis and organ dysfunction. Vascular injury to capillary endothelium may be the causative factor for capillary leak. ${ }^{14}$ Edema has been reported from $37 \%$ to $60 \%$ in two studies from India. ${ }^{8}, 13$ Our study reported edema in $77 \%$ of the patients. ${ }^{4}$ Similarly, hypoalbuminemia was seen in $82.6 \%$ of our patients while kumar et al reported Hypoalbunimenia in $54 \%$ of the patients. ${ }^{15}$

Lymphadenopathy in scrub typhus patient is regional to begin with, large and tender lymph nodes occurs at the site of bite and may lead to generalised lymphadenopathy, Lymphadenopathy was seen in $84.6 \%$ of children in our study, similarly Kumar et al reported in $37 \%$ and Silva et al from Sri Lanka reported in $60 \%$ of children. ${ }^{15,16}$

Eschar was seen in $11.8 \%$ of our study population, similar to $11 \%$ by Kumar et al and $14 \%$ by Somashekar at al. ${ }^{15,17}$ A recent study (2019) in India by Bal et al in 201 children showed eschar in $17.9 \%$ cases. ${ }^{18}$ Studies from different countries have shown variation in presence of eschar ranging from $50-80 \%$ cases. ${ }^{9} 19$ Presence of eschar in a child with fever, thrombocytopenia and capillary leak may help to differentiate from Dengue infection. ${ }^{11,13}$

Complications were studied in patients with scrub typhus. Myocarditis (67.3\%), hypoalbuminemia (82.6\%) and AKI $(65.4 \%)$ were three major complications in our study. Pathak et al reported myocarditis (72.4\%), hypoalbuminemia $(71.1 \%)$, severe thrombocytopenia $(22.4 \%)$ as major complications.' Myocarditis with cardiogenic shock at presentation was the most common complication $(34 \%)$ seen by Kumar et $a^{15}$ Majority of children with myocarditis also had shock at presentation and required diuretic therapy, fluid restriction, and vasoactive support. All patients with AKI improved with conservative management and none required dialysis. Intravascular fluid depletion and MODS could be the cause of AKI. Meningoencephalitis was seen in $15.4 \%$ of the admitted patients. $26 \%$ had meningoencephalitis in study by Vishwanathan et al from India and Kim et al reported in $7.3 \%$ of children. ${ }^{20,21}$

Our study found open defecation in field or movement of children in forest area/rice field as the risk factor for scrub typhus disease. 28 (54\%) children confirmed open defecation, while outdoor play in nearby forest and area with heavy vegetation was seen in $6(11.5 \%)$ children.
Study by Gautam et al demonstrated houses near grassland and working in filed to be significantly associated with scrub typhus disease. ${ }^{5}$ Factors that may have resulted in increased risk were identified in some of the outbreaks, includes patients squatting when relieving themselves in the bushes. ${ }^{22}$

Cochrane review (2018) concluded that tetracycline, doxycycline, azithromycin, and rifampicin are effective drugs available for the treatment of scrub typhus and suggested that there may be little or no difference between tetracycline, doxycycline, and azithromycin as treatment options. The review also found that there were few treatment failures with the above-mentioned drugs. ${ }^{23}$ Majority of children in our study were treated with doxycycline and showed a good clinical response. Previous studies have also shown a similar clinical response to doxycycline. ${ }^{11,15}$ Few patients were treated with doxycycline as well as Azithromycin. Four children could not be salvaged even after treatment. Mortality rate in our study $(7.7 \%)$ was similar to study by Rathi et al $(9 \%)$ and lower than Kamarasu et al $(15 \%){ }^{24,25}$

\section{Limitations of the study}

The present study has some limitations. As the study was conducted in a tertiary care referral hospital, the present findings may not reflect the exact burden of the disease in the community.

\section{CONCLUSION}

High index of suspicion in febrile children along with presence of eschar, lymphadenopathy and capillary leak is the hallmark of this disease. Prompt diagnosis will prevent from severe complications like Myocarditis, AKI, and Meningoencephalitis.

\section{ACKNOWLEDGEMENT}

The authors would like to thanks the Residents at Department of paediatrics.

\section{REFERENCES}

1. Koh GC, Maude RJ, Paris DH, Newton PN and Blacksell SD. Diagnosis of scrub typhus. Am J Trop Med Hyg. 2010;82(3): 368-370.

https://doi.org/10.4269/ajtmh.2010.09-0233

2. Mahajan SK, Rolain JM, Sankhyan N, Kaushal RK and Raoult D. Pediatric scrub typhus in Indian Himalayas. Indian Journal of Pediatrics. 2008; 75(9), 947-949.

https://doi.org/10.1007/s12098-008-0198-z

3. Luce-Fedrow A, Lehman ML, Kelly DJ, Mullins K and Maina A. A Review of Scrub Typhus (Orientia tsutsugamushi and Related 
Organisms): Then, Now, and Tomorrow. Tropical medicine and infectious disease. 2018; 3(1):3-4.

https://doi.org/10.3390/tropicalmed3010008

4. Murdoch DR, Woods CW, Zimmerman MD, Dull PM, Belbase RH and Keenan AJ. The etiology of febrile illness in adults presenting to Patan hospital in Kathmandu, Nepal. Am J Trop Med Hyg. 2004; 70(6):670-675.

https://doi.org/10.4269/ajtmh.2004.70.670

5. Gautam R, Parajuli K and Sherchand JB. Epidemiology, Risk Factors and Seasonal Variation of Scrub Typhus Fever in Central Nepal. Tropical medicine and infectious disease. 2019; 4(1):27.

https://doi.org/10.3390/tropicalmed4010027

6. Sivarajan S, Shivalli S, Bhuyan D, Mawlong M and Barman R. Clinical and paraclinical profile, and predictors of outcome in 90 cases of scrub typhus, Meghalaya, India. Infect Dis Poverty. 2016;5;5(1):91.

https://doi.org/10.1186/s40249-016-0186-x

7. Huang CT, Chi H, Lee HC, Chiu NC and Huang FY. Scrub typhus in children in a teaching hospital in eastern Taiwan, 2000-2005. Southeast Asian J Trop Med Public Health. 2009;7; 40(4):789-794.

8. Kumar Bhat N, Dhar M, Mittal G, Shirazi N, Rawat A, Prakash Kalra B, et al. Scrub typhus in children at a tertiary hospital in north India: clinical profile and complications. Iran J Pediatr. 2014;8;24(4):387-392.

9. Pathak S, Chaudhary N, Dhakal P, Shakya D, Dhungel P, Neupane G, et al. Clinical profile, complication, and outcome of scrub typhus in children: A hospital based observational study in central Nepal. PLoS One. 2019;8;13;14(8):e0220905.

https://doi.org/10.1371/journal.pone.0220905

10. Karki KB, Acharya BP, Dhimal M, Aryal KK, Sharma GN and Khanal P. Descriptive Epidemiology of Scrub Typhus in Nepal. Kathmandu, Nepal, 2017. Nepal Health Research Council (NHRC).2017; 14-15.

11. Behera B, Biswal M, Das RR, Dey A, Jena J, Dhal S, et al. Clinico-epidemiological analysis of scrub typhus in hospitalised patients presenting with acute undifferentiated febrile illness: A hospital-based study from Eastern India. Indian J Med Microbiol. 2019; 37(2):278-280.

https://doi.org/10.4103/ijmm.IJMM_19_147

12. Sirisanthana V, Puthanakit $T$ and Sirisanthana T. Epidemiologic, clinical and laboratory features of scrub typhus in thirty Thai children. Pediatr Infect Dis J. 2003; 22(4):341-345.

https://doi.org/10.1097/01.inf.0000059400.23448.57

13. Palanivel S, Nedunchelian K, Poovazhagi V, Raghunadan R and Ramachandran P. Clinical profile of scrub typhus in children. Indian J Pediatr. 2012;11;79(11):1459-1462. https://doi.org/10.1007/s12098-012-0721-0

14. Baranwal A and.Subramani S. Scrub Typhus: A literature Review,
Journal of Pediatric Critical Care. 2019 ;6; 4: 24-27.

https://doi.org/10.21304/2019.0604.00515

15. Kumar M, Krishnamurthy S, Delhikumar CG, Narayanan $P$, Biswal $\mathrm{N}$ and Srinivasan S. Scrub typhus in children at a tertiary hospital in southern India: clinical profile and complications. J Infect Public Health. 2012; 3; 5(1):82-88.

https://doi.org/10.1016/j.jiph.2011.11.001

16. De Silva N, Wijesundara S, Liyanapathirana $\mathrm{V}$, Thevanesam $\mathrm{V}$ and Stenos J. Scrub Typhus among Pediatric Patients in Dambadeniya: A Base Hospital in Sri Lanka. Am J Trop Med Hyg. 2012;8;87(2):342-344.

https://doi.org/10.4269/ajtmh.2012.12-0170

17. Somashekar HR, Moses PD, Pavithran S, Mathew LG, Agarwal I and Rolain JM. Magnitude and features of scrub typhus and spotted fever in children in India. J Trop Pediatr. 2006;6;52(3):228-229.

https://doi.org/10.1093/tropej/fmi096

18. Bal M, Mohanta MP, Sahu S, Dwibedi B, Pati S and Ranjit M. Profile of Pediatric Scrub Typhus in Odisha, India. Indian Pediatrics2019;4;56(4):304-306. https://doi.org/10.1007/s13312-019-1519-1

19. Lee CS, Min IS, Hwang JH, Kwon KS and Lee HB. Clinical significance of hypoalbuminemia in outcome of patients with scrub typhus. BMC Infect Dis. 2010;7;21;10:216. https://doi.org/10.1186/1471-2334-10-216

20. Viswanathan S, Muthu V, Iqbal N, Remalayam B and George T. Scrub typhus meningitis in South India--a retrospective study. PLoS One. 2013;6;8(6):e 66595. https://doi.org/10.1371/journal.pone.0066595

21. Kim DM, Chung JH, Yun NR, Kim SW, Lee JY and Han MA. Scrub typhus meningitis or meningoencephalitis. Am J Trop Med Hyg. 2013;12;89(6):1206-1211. https://doi.org/10.4269/ajtmh.13-0224

22. Singh SI, Devi KP, Tilotama R, Ningombam S, Gopalkrishna Y, Singh TB, et al. An outbreak of scrub typhus in Bishnupur district of Manipur, India, 2007. Trop Doct. $2010 ; 40(3): 169-170$. https://doi.org/10.1258/td.2010.090468

23. El Sayed I, Liu Q, Wee I and Hine P. Antibiotics for treating scrub typhus. Cochrane Database of Systematic Reviews 2018, Issue 9. Art. No.: CD002150. https://doi.org/10.1002/14651858.CD002150.pub2

24. Rathi NB, Rathi AN, Goodman MH and Aghai ZH. Rickettsial diseases in central India: proposed clinical scoring system for early detection of spotted fever. Indian Pediatr. 2011;11;48(11):867-872. https://doi.org/10.1007/s13312-011-0141-7

25. Kamarasu K, Malathi M, Rajagopal V, Subramani K, Jagadeeshramasamy $D$ and Mathai E. Serological evidence for wide distribution of spotted fevers \& typhus fever in Tamil Nadu. Indian J Med Res. 2007;8;126(2):128-130.

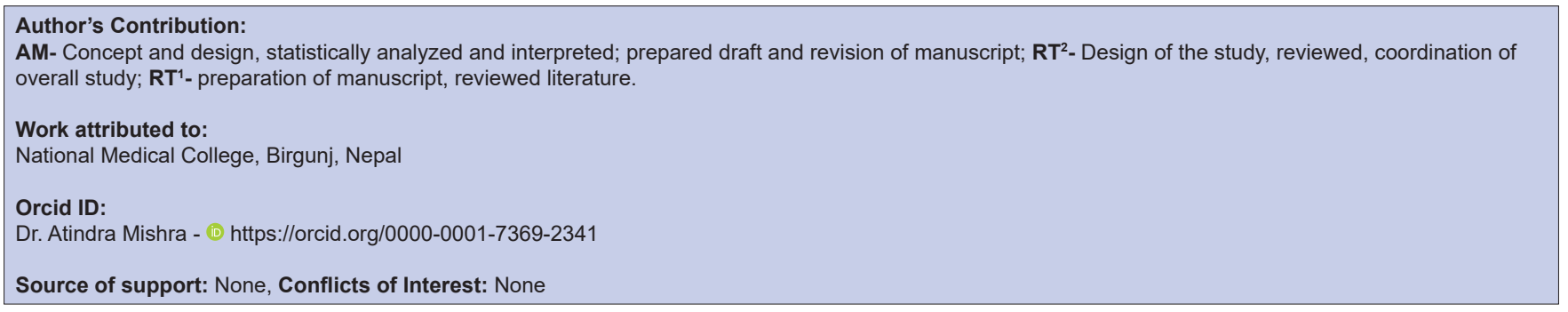

